\title{
Catalytic radical generation of $\pi$-allylpalladium complexes
}

\author{
Huan-Ming Huang, Maximilian Koy, Eloisa Serrano, Philipp Miro Pflüger, J. Luca Schwarz, and Frank Glorius* \\ Organisch-Chemisches Institut, Westfälische Wilhelms-Universität Münster, Corrensstraße 40, 48149 \\ Münster, Germany \\ *Correspondence author. Email: glorius@uni-muenster.de
}

\begin{abstract}
Transition-metal-catalyzed allylic substitution is one of the most powerful and frequently used methods in organic synthesis. In particular, palladium-catalyzed allylic functionalization has become a well-established strategy to construct carbon-carbon or carbon-heteroatom bonds, and its utility has been demonstrated in natural product synthesis, drug discovery and materials science. Several methods have been developed to generate $\pi$-allylpalladium complexes through ionic mechanisms; however, these methods typically require either prefunctionalized starting materials or stoichiometric oxidants, which naturally limits their scope. Here we show a radical approach for the generation of $\pi$-allylpalladium complexes by employing $N$-hydroxyphthalimide esters as bifunctional reagents in combination with 1,3-dienes. Using this strategy, we report the 1,4-aminoalkylation of dienes. The remarkable scope and functional group tolerance of this redox-neutral and mild protocol was demonstrated across $>60$ examples. The utility of this strategy was further demonstrated in radical cascade reactions and in the late-stage modification of drugs and natural products.
\end{abstract}

\section{Introduction}

The development of more selective, sustainable and atom-economical synthetic strategies is a fundamental goal of organic synthesis. ${ }^{1-3}$ Breakthroughs in this area serve to drive innovation and accelerate discovery in all sectors of industry. ${ }^{4}$ For example, the development of transition-metal-catalyzed allylic substitution reactions has enabled significant advances in synthetic, medicinal and materials science. ${ }^{5-8}$ However, despite being developed several decades ago, several challenges concerning the reactivity and sustainability of this strategy still remain.

Palladium catalyzed allylic substitution reaction is one of the most versatile and frequently used methods in nucleophilic allylic functionlization. ${ }^{5-7}$ Currently, the most important intermediate in this transformation, 
the $\pi$-allylpalladium complex, can be generated by four main methods with ionic mechanisms (Fig. 1a). ${ }^{9,10}$ The most common and straightforward way (the Tsuji-Trost reaction) proceeds via the $\mathrm{C}-\mathrm{X}$ bond cleavage of a prefunctionalized allyl(pseudo)halide electrophile. ${ }^{5}$ More recently, elegant methods pioneered by White ${ }^{11}$ and Shi ${ }^{12}$ for the catalytic generation of $\pi$-allylpalladium complexes directly from allylic $\mathrm{C}-\mathrm{H}$ bonds have been developed. However, exogenous stoichiometric oxidants are required and the reaction conditions are relatively harsh. Alternatively, 1,3-dienes have also been used as atom-economic precursors for the generation of $\pi$-allylpalladium complexes via nucleopalladation. However, stoichiometric amounts of oxidant are again necessary to regenerate the active $\operatorname{Pd}(\mathrm{II})$ catalyst in this strategy. ${ }^{13}$ Additionally, $\pi$-allylpalladium complexes can be formed through oxidative addition of a $\operatorname{Pd}(0)$ species into an aryl or vinyl halide followed by migratory insertion into a 1,3-diene. ${ }^{14-17}$ Methods for the generation of $\pi$-allylpalladium complexes from alkyl halides or analogous electrophiles using $\mathrm{Pd}(0)$ (for the formation of $\mathrm{C}\left(\mathrm{sp}^{3}\right)-\mathrm{C}\left(\mathrm{sp}^{3}\right)$ bonds) have not been realized due to the reluctance of alkyl halides to undergo oxidative addition and the fast rate of $\beta$-hydride elimination of Pd-alkyl species. ${ }^{18}$ However, recent breakthroughs in photoredox chemistry, ${ }^{19-21}$ have shown that photoexcited palladium catalysts can be used to generate open-shell species from alkyl halide precursors. ${ }^{22}$ Thus, we questioned whether $\pi$-allylpalladium complexes could also be generated through a single-electron transfer (SET) mechanism to overcome the aforementioned limitations. Here we envisioned that a photoexcited $\operatorname{Pd}(0)$ complex could be used to reduce an alkyl radical precursor to generate an alkyl radical and a Pd(I) species. The newly-formed alkyl radical could then be trapped by a feedstock diene, such as 1,3-butadiene, to afford a radical intermediate that could readily after recombine with the $\mathrm{Pd}(\mathrm{I})$ species to generate the desired $\pi$-allylpalladium complex (Fig. 1b).

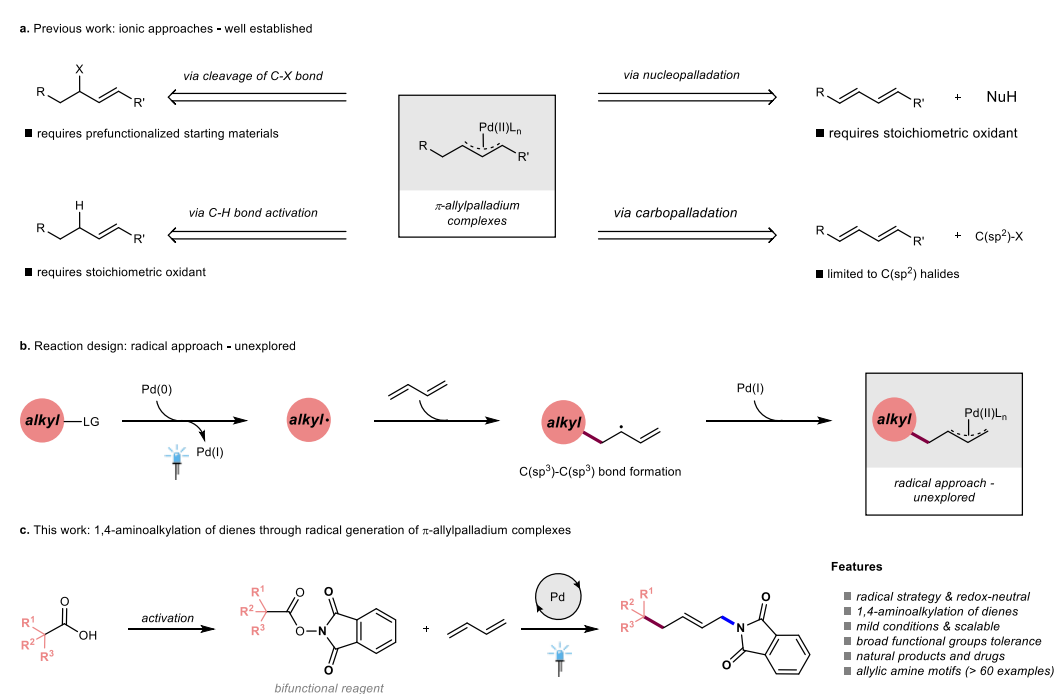

Fig. 1 | Development of a radical strategy for the generation of $\pi$-allylpalladium complexes and its 
application in the 1,4-aminoalkylation of dienes. (a) Previous work using ionic approaches, (b) conceptual radical approach, (c) this work.

Inspired by the numerous elegant reports of radical decarboxylation using $N$-hydroxyphthalimide (NHP) esters derived from inexpensive and abundant aliphatic carboxylic acids, ${ }^{23-32}$ especially, Shang \& $\mathrm{Fu}^{33,34}$ and Glorius $^{35}$ have reported excellent examples of palladium-catalyzed decarboxylative Heck reaction 33,35 and desaturation ${ }^{34}$ mediated by visible light, we hypothesized that $\pi$-allylpalladium complexes could be generated by merging abundant NHP esters and feedstock dienes under palladium catalysis. However, it was uncertain at the outset of our studies whether NHP esters could be used as bifunctional reagents (both radical precursor and nucleophile) in this approach,36,37 or if the stereo- and regioselectivity of the diene difunctionalization could be controlled in such a radical process. ${ }^{15}$

Herein, we present the generation of $\pi$-allylpalladium complexes through a radical approach mediated by visible light. The synthetic potential of this strategy was demonstrated through the 1,4-aminoalkylation of dienes (Fig. 1c). The obtained allylic amine products represent fundamentally important building blocks in synthetic chemistry could be readily diversified. ${ }^{6}$

\section{Results}

Reaction development. Our studies began by reacting NHP ester (1a) and butadiene (2a) with catalytic amount of $\mathrm{Pd}\left(\mathrm{PPh}_{3}\right)_{4}$ under blue LEDs irradiation. After $24 \mathrm{~h}$, we were delighted to find that the 1,4-aminoalkylation product (3) was formed in good yield, with excellent diastereoselectivity ( $>95: 5 \mathrm{dr}$ ) and complete regioselectivity ( $>$ 20:1 rr) (Table 1, entry 1). Then a survey of solvents, palladium sources and ligands was conducted, the 1,4-aminoalkylation product (3) was obtained in $83 \%$ isolated yield only using $7 \mathrm{~mol} \% \mathrm{Pd}\left(\mathrm{PPh}_{3}\right)_{4}$, with excellent stereoselectivity (> 95:5 E/Z) and complete regioselectivity (> 20:1 rr). Control experiments omitting each individual reaction component highlighted the importance of both the palladium catalyst and visible light to form the desired 1,4-aminoalkylation product ( See Supplementary Table 1). Additionally, assessment of the reaction-condition-based sensitivity was performed, which indicated that the reaction is only sensitive towards air and temperature ( See Supplementary Method 8).38

Table 1 | Optimization of radical decarboxylative 1,4-aminoalkylation of butadiene ${ }^{a}$ 


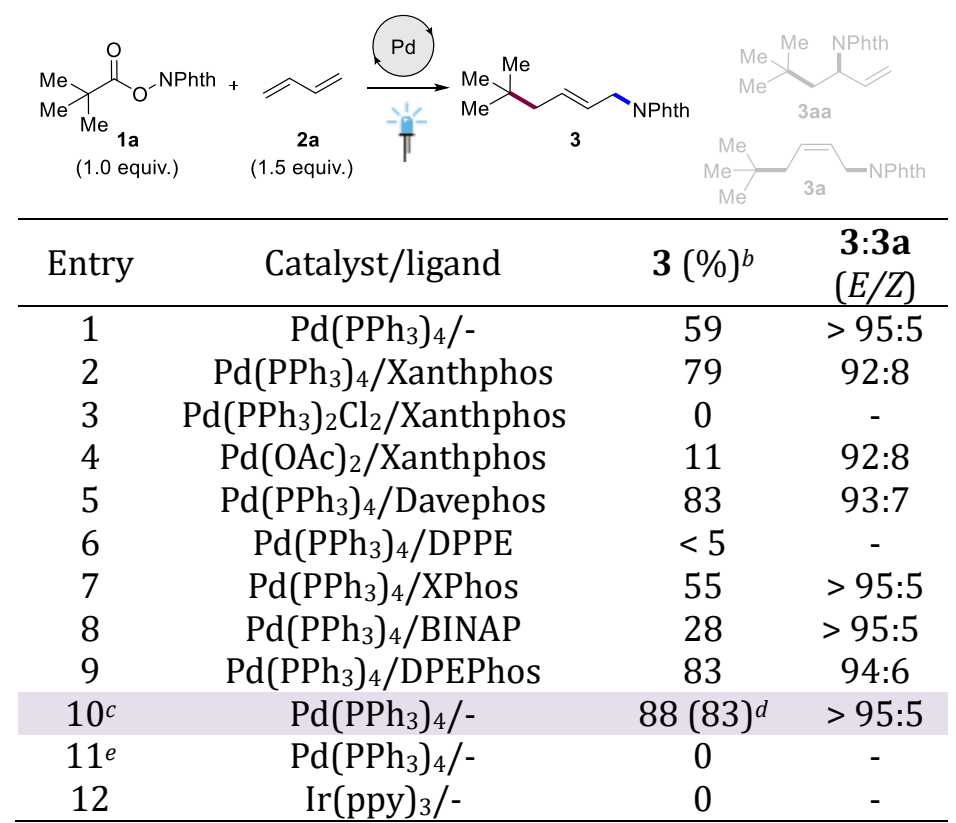

${ }^{a}$ Reaction conditions: NHP ester 1a $(0.2 \mathrm{mmol})$, catalyst $(5 \mathrm{~mol} \%)$, ligand $(6 \mathrm{~mol} \%)$, butadiene 2a $(0.3$ mmol, $2 \mathrm{M}$ in THF, $0.15 \mathrm{~mL}$ ), DMA (0.85 mL), RT, blue LEDs (5W, $455 \mathrm{~nm}), 24 \mathrm{~h}$, under argon. Diastereomeric and regioselective ratios were determined by ${ }^{1} \mathrm{H}$ NMR spectroscopy of crude product mixtures and regioselectivity of $(\mathbf{3 + 3 a}): 3 a a$ is $>20: 1$ in all the case. ${ }^{b}$ Yield was determined by ${ }^{1} \mathrm{H}$ NMR spectroscopy analysis with 1,3,5-trimethoxybezene as internal standard. $c 7 \mathrm{~mol} \% \mathrm{Pd}\left(\mathrm{PPh}_{3}\right)_{4}$ was added. $d$ Isolated yields. ${ }^{e}$ Without blue LEDs at room temperature, $50{ }^{\circ} \mathrm{C}$ or $100{ }^{\circ} \mathrm{C}$. Highlighted entry: optimal conditions found.

Reaction scope. We then began to investigate the substrate scope with respect to the two reaction partners (Fig. 2). First, we explored NHP esters derived from alkyl carboxylic acids and found the scope to be remarkably broad. In general, tertiary, secondary and primary acids were all compatible and the corresponding products could be obtained in good to excellent yields. 15 different tertiary alkyl carboxylic acids were investigated in order to construct (all-carbon) quaternary centers. Acyclic (3-6, 15), cyclic including cyclopropyl $(\mathbf{7}, \mathbf{8})$, adamantyl $(\mathbf{9})$, noradamantyl $(\mathbf{1 0})$, cyclohexyl $(\mathbf{1 1}, \mathbf{1 6})$, heterocyclic $(\mathbf{1 2})$, oxetanyl (13) and bicyclo[2.2.2] octanyl (14) were all compatible in this radical cross-coupling reaction. In all the cases, the products were obtained in excellent yields, with complete stereo- and regiocontrol. Various secondary and primary aliphatic carboxylic acids were also tested. Interestingly, whilst product $\mathbf{1 7}$ was obtained in $86 \%$ yield and $86: 14 E / Z$ under our standard reaction conditions, the stereoselectivity could be restored (> 95:5 E/Z) by using BINAP as a ligand. These results are consistent with a scenario in which show that both syn- and anti-allylic palladium complexes have been attacked by nucleophiles through allylic amination without additional BINAP ligand. Thus both $\mathrm{E}$ and $\mathrm{Z}$ isomers of product $\mathbf{1 7}$ were obtained. However, when BINAP is used in the standard condition, only E isomer of $\mathbf{1 7}$ was observed. If a bidentate 
ligand (BINAP) is employed instead of a monodentate ligand $\left(\mathrm{PPh}_{3}\right)$, due to the kinetic and thermodynamic chelate effect, the BINAP-palladium metal interaction is less likely to break compared to the $\mathrm{PPh}_{3}$-palladium metal interaction. So $\eta^{3}-\eta^{1}-\eta^{3}$ isomerization will be more difficult due to steric reasons when BINAP was employed in the standard condition. This could be the possible reason for higher selectivity when BINAP was employed in this system. Again, cyclic $(\mathbf{1 9}, \mathbf{2 1}, \mathbf{2 3})$, heterocyclic $(\mathbf{1 8}, \mathbf{1 9})$ and acyclic (24-29), difluorinated (20), indane (22) moieties smoothly participated in the cross-coupling reaction. A broad range of functional groups such as trifluoromethyl $(\mathbf{5})$, amides $(\mathbf{1 2}, \mathbf{1 6}, \mathbf{1 8})$, ethers $(\mathbf{1 3}, \mathbf{1 7})$ and esters $(\mathbf{6}$, 15) were found to be compatible. Next, the scope of the dienes was evaluated. Interestingly, the desired allylic amine products could not be obtained using our standard conditions. However, the desired reactivity could be completely reestablished simply by using DPEPhos as a ligand. Similar phenomenon has been reported by Beller and co-workers in palladium catalyzed hydroamination of 1,3-dienes. ${ }^{39}$ Commercially available DPEphos with large bite angle could react with palladium catalyst to form a stable palladiumphosphine complex. The complex has played a crucial role in the reactivity and selectivity during the allylic amination step. Using these conditions, simple feedstock dienes such as 2,3-dimethyl-1,3-butadiene (30, 31), isoprene (32) and 1,3-cyclohexadiene (33-35) were all converted into the desired products in moderate yields. Finally, several substituted 1,3-dienes (36-44) were evaluated and the corresponding allylic amine motifs could be obtained in moderate to excellent yields with excellent stereo- and regioselectivities (mostly, $>95: 5 E / Z$ and $>20: 1 \mathrm{rr}$ ). The relative stereochemistry of the radical cross-coupling product (20) was confirmed by X-ray crystallography. In addition, the synthetic utility of our radical approach was further demonstrated in gram-scale reactions ( 3 and $\mathbf{9}$ ) without a notable decrease in the efficiency. 


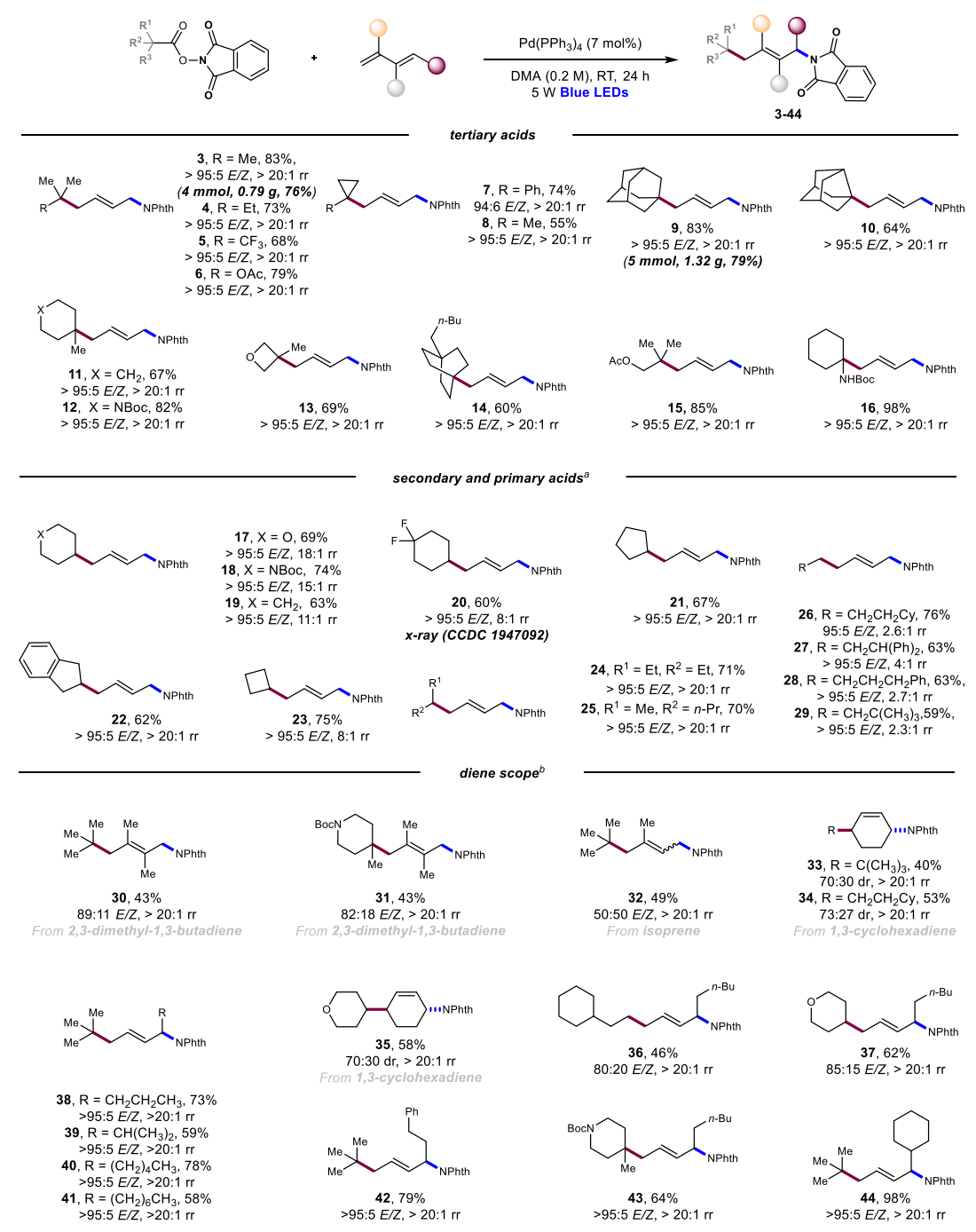

Fig. 2 | Scope of palladium catalyzed 1,4-aminoalkylation of 1,3-dienes. Reaction conditions: NHP ester 1 (0.2 mmol), Pd( $\left.\mathrm{PPh}_{3}\right)_{4}(7 \mathrm{~mol} \%)$, butadiene 2a (0.3 mmol, $2 \mathrm{M}$ in THF, $\left.0.15 \mathrm{~mL}\right)$, DMA (0.85 mL), RT, blue LEDs ( $5 \mathrm{~W}, 455 \mathrm{~nm}), 24 \mathrm{~h}$, under argon. Isolated yields are shown. Diastereomeric ratios and regioselectivity were determined by ${ }^{1} \mathrm{H}$ NMR spectroscopy of crude product mixtures. ${ }^{a} 9$ mol\% BINAP was added. ${ }^{b} 9$ mol\% DPEphos and the corresponding substituted 1,3-diene (3.0 equiv.) were used.

Modification of complex architectures. To showcase the robustness and utility of the developed transformation, several NHP esters derived from natural products and drugs were also converted into their corresponding 1,4-aminoalkylation products (Fig. 3). Aside from amino acid derived precursors, several drugs and natural product derivatives (51-59) were also examined. Pleasingly, structurally complex architectures bearing multiple functional groups such as dehydrocholic acid (51), lithocholic acid $(\mathbf{5 2}, \mathbf{5 3})$, oleanolic acid (54), abietic acid (55), dehydroabietic acid (56), gemfibrozil (58), and ciprofibrate (59) were all tolerated. The modified complex products were obtained in good to excellent yields and with high selectivity. Good functional group compatibility was also established with ketone (51), esters (52), 
unprotected alcohols $(\mathbf{5 3}, \mathbf{5 4})$, alkene $(\mathbf{5 4})$, conjugated diene $(\mathbf{5 6})$, ethers $(\mathbf{5 8}, \mathbf{5 9})$ and halide $(\mathbf{5 9})$.

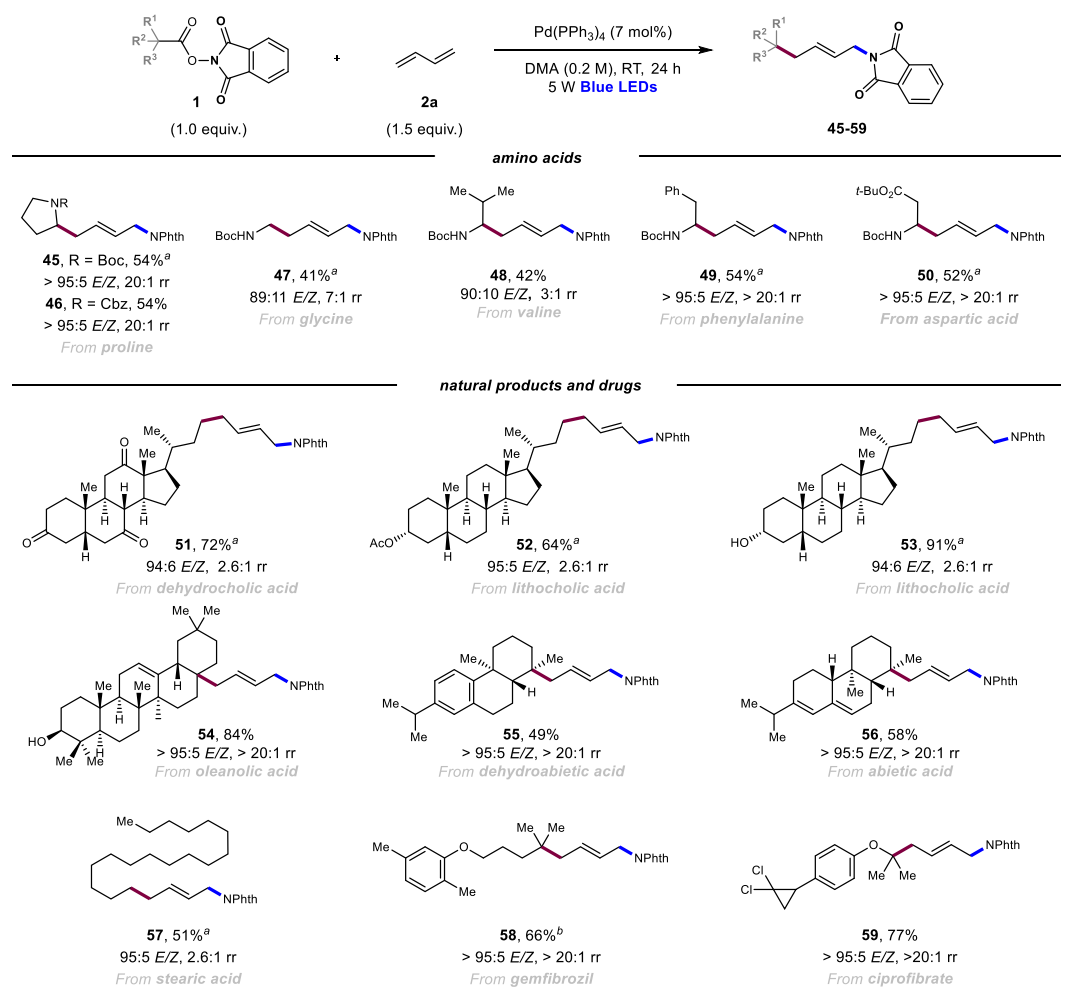

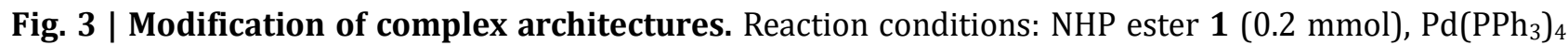
(7 mol\%), butadiene 2a (0.3 mmol, $2 \mathrm{M}$ in THF, $0.15 \mathrm{~mL}$ ), DMA (0.85 mL), RT, blue LEDs ( $5 \mathrm{~W}, 455 \mathrm{~nm}), 24 \mathrm{~h}$, under argon. Isolated yields are shown. Diastereomeric and regioselectivity ratios were determined by ${ }^{1} \mathrm{H}$ NMR spectroscopy of crude product mixtures. ${ }^{a} 9$ mol\% BINAP was added. ${ }^{b} 10 \mathrm{~mol} \% \mathrm{Pd}\left(\mathrm{PPh}_{3}\right)_{4}$ was added.

Radical cascade reactions and synthetic application. Cascade reactions are a powerful tool for the rapid generation of complex architectures and are frequently used in the synthesis of natural products and complex drug candidates. ${ }^{21,40}$ We questioned whether cascade reactions involving the radical decarboxylative generation of $\pi$-allylpalladium complexes could also be developed. To demonstrate this concept, the NHP ester derived from cis-pinonic acid was converted into the complex scaffold $\mathbf{6 0}$, containing a ketone, terminal and internal alkenes and a protected amine, under our standard conditions with complete stereoselectivity. Additionally, several related scaffolds (61-64) were also obtained in moderate yields and with excellent stereo- and regioselectivities (Fig. 4a). Here, we propose that the key tertiary radical intermediate II is generated by the fragmentation of intermediate I. $^{41}$ Gratifyingly, radical cyclisation/cross-coupling cascade reaction was also developed by reacting butadiene with the NHP ester derived from citronellic acid, and the complex architecture 65 was obtained in good yield (Fig. 4b). Finally, three post-catalysis transformations of product 9 (dihydroxylation, hydrogenation, phthalimide deprotection) were performed to afford the corresponding products in excellent yields (Fig. 4c). 


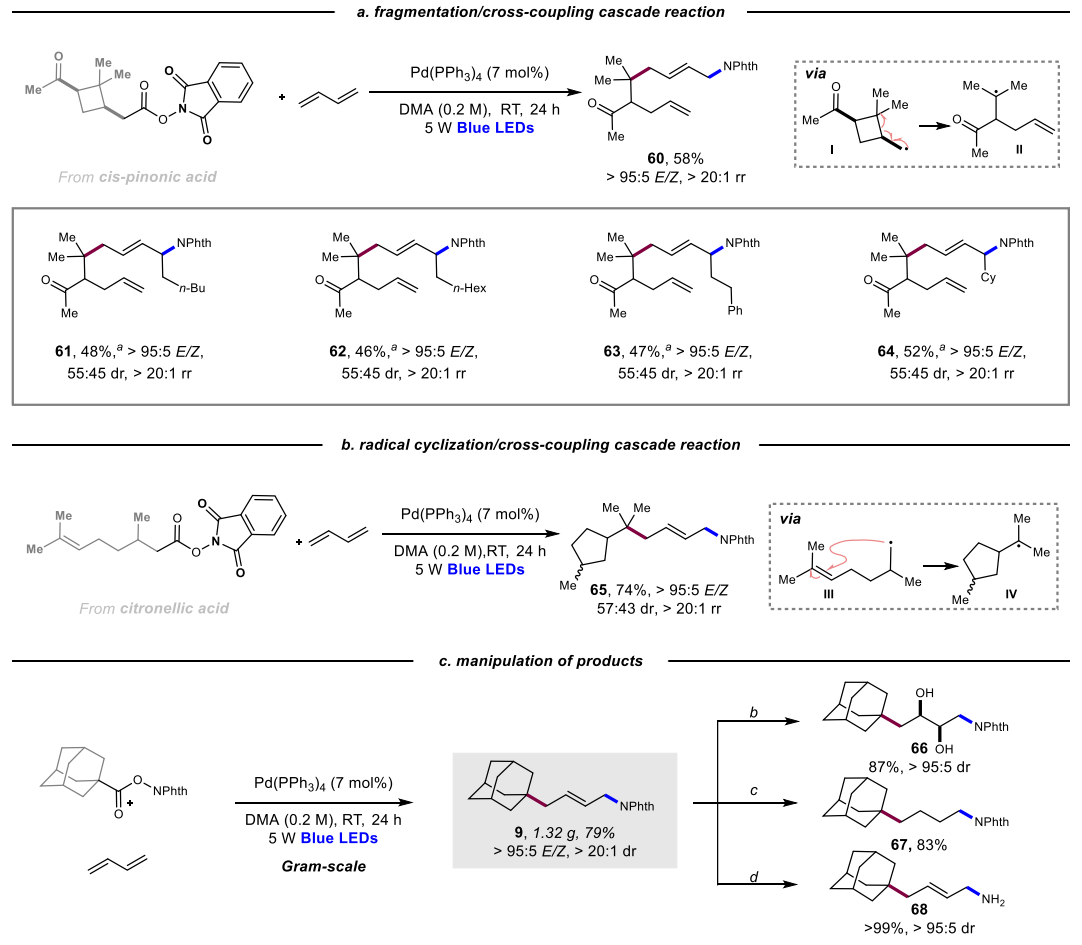

Fig. 4 | Cascade reactions and manipulation of allylic amines. (a) Fragmentation/cross-coupling cascade reaction. (b) Radical cyclisation/cross-coupling cascade reaction. (c) Manipulation of products. ${ }^{a} 9$ mol\% DPEphos and the corresponding substituted 1,3-diene (3.0 equiv.) were used. ${ }^{b} \mathrm{~K}_{2} \mathrm{OsO}_{4} \cdot 2 \mathrm{H}_{2} \mathrm{O}$ (5 mol\%), NMO (3 equiv.), $t$-BuOH:H $\mathrm{H}_{2} \mathrm{O}: \mathrm{THF}=1: 1: 1, \mathrm{RT}, 20$ h. ${ }^{c}$ Pd/C (10 mol\%), $\mathrm{H}_{2}(10 \mathrm{bar}), \mathrm{EtOAc}, \mathrm{RT}, 24$ h. ${ }^{d}$ $\mathrm{NH}_{2} \mathrm{NH}_{2} \cdot \mathrm{H}_{2} \mathrm{O}$ (3.3 equiv.), $\mathrm{MeOH}(0.3 \mathrm{M}$ ), RT, $40 \mathrm{~min}$, then 5\% HCl, RT, $16 \mathrm{~h}$.

Preliminary Mechanistic Investigations. UV-vis absorption experiments confirmed that, of the starting materials, $\mathrm{Pd}\left(\mathrm{PPh}_{3}\right)_{4}$ is the only light absorbing species in the reaction system. Stern-Volmer studies of $\mathrm{Pd}\left(\mathrm{PPh}_{3}\right)_{4}$ with NHP ester 1a support that a photoexcited $\mathrm{Pd}(0)$ species could reduce 1a through single electron-transfer (See Supplementary Figure 7). These results are consistent with previous reports by Shang and $\mathrm{Fu}^{33,34}$ and our group ${ }^{35}$. When TEMPO was added to the standard reaction conditions as a radical inhibitor, all formation of the desired product was suppressed and a TEMPO adduct was detected by high-resolution mass spectrometry (HRMS). To provide further evidence of a radical process, a radical probe experiment was performed, which afforded the expected ring-opening products (69 and 69') in 46\% and 13\% yields, respectively (Fig. 5a). Furthermore, the structure of radical trap $\mathbf{7 0}$ was confirmed by EPR spectrometry, which strongly supports the proposed formation of a tert-butyl alkyl radical (Fig. 5b, See Supplementary Method 7.4). The EPR signal was only observable after irradiation with blue LEDs. This is in line with previous studies by Shang and $\mathrm{Fu},{ }^{34}$ and indicates that SET from excited $\mathrm{Pd}(0)$ species to NHP 
ester 1a occurs only after light irradiation to likely form a hybrid alkyl Pd(I) radical species VII.33-35 Additionally, X-ray photoelectron spectroscopy (XPS) measurements were performed and indicate the presence of three distinct $\operatorname{Pd}$ oxidation states, namely $\operatorname{Pd}(0), \operatorname{Pd}(\mathrm{I})$ and $\operatorname{Pd}(\mathrm{II})$, which suggests that $\operatorname{Pd}(\mathrm{I})$ species may be involved in the process. Based on these preliminary mechanistic studies and supported by recent reports by Gervogyan, ${ }^{42-48}$ Shang and $\mathrm{Fu},{ }^{34,49,50} \mathrm{Yu},{ }^{51}$ and Rueping52, we proposed that a hybrid alkyl $\mathrm{Pd}(\mathrm{I})$ radical species VII is generated from the NHP ester 1a by SET from the photoexcited palladium catalyst. ${ }^{33-35}$ Recent developments in alkyl Heck-type reactions have shown that the hybrid alkyl Pd(I) radical species could be generated by visible light and can subsequently react efficiently with styrene-type substrates.33,35,43,45,52-54 Based on the seminal work by Minisci, which shows that the rate of alkyl radical addition to butadiene is faster than styrene in solution, ${ }^{55}$ we propose that butadiene $2 \mathbf{a}$ may react with the hybrid alkyl Pd(I) radical species VII to produce the putative hybrid allylic $\mathrm{Pd}(\mathrm{I})$ radical species VIII. Alernatively, butadiene 2a may pre-associate to the Pd species, which would accelerate the rate of alkyl radical addition to $\mathbf{2 a}$ and the recombination of the allylic radical with the $\mathrm{Pd}(\mathrm{I})$ radical species ${ }^{56,57}$ (See Supplementary Method 7.11). According to the result by ESI-MS analysis (Fig. 5c), the possible palladium(II) complex (71 or 71' or 71') has been generated. Finally, it is proposed that the phthalimide unit (generated in the first step and usually treated as a waste byproduct ${ }^{23-35}$ ) attacks the proposed $\pi$-allylpalladium complex IX to selectively afford the product $\mathbf{3}$ and regenerate the $\operatorname{Pd}(0)$ catalyst. Additionally, a similar $\pi$-allylpalladium complex ${ }^{58}$ has been pre-formed and subjected to our reaction condition, the corresponding allylic amine was obtained in $69 \%$ isolated yield which supports our proposed allylic amination step and is consistent to the previous work reported by Liu ${ }^{59}$ and White ${ }^{60}$. An alternative chain reaction seems unlikely due to the mismatched redox potentials of the NHP ester $1 \mathrm{a}\left(\mathrm{E}_{1 / 2^{\text {red,calc }}}=-1.24 \mathrm{~V}\right.$ vs SCE$)$ with tert-butyl radical $\left(E_{1 / 2^{\text {red,calc }}}=-0.21 \mathrm{~V}\right.$ vs $\left.S C E\right)$, allylic radical $\left(E_{1 / 2}^{\text {red,calc }}=+0.62 \mathrm{~V}\right.$ vs SCE $)$. 


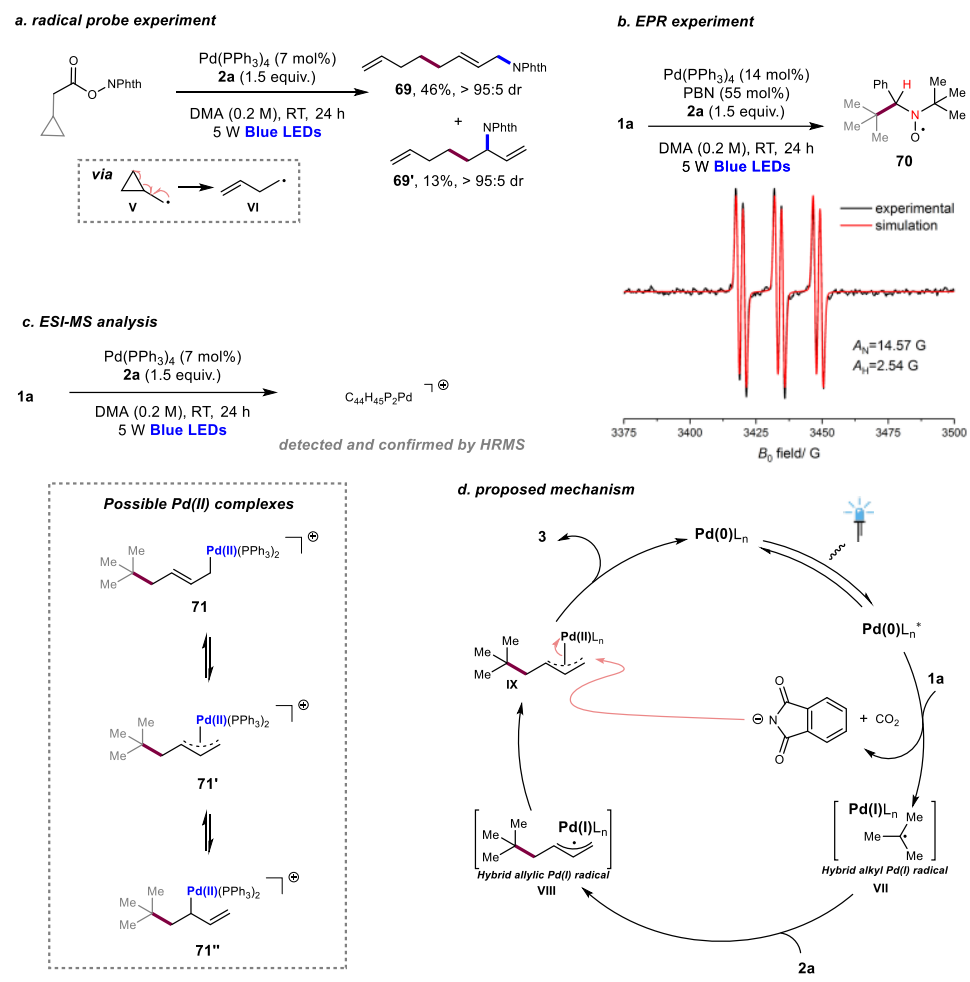

Fig. 5 | Preliminary mechanistic studies and proposed mechanism. (a) Radical probe. (b) Electron Paramagnetic Resonance (EPR). (c) Electrospray ionization mass spectrometry (ESI-MS). (d) Proposed mechanism.

\section{Conclusion}

In summary, we have established a general strategy for the generation of $\pi$-allylpalladium complexes through a radical mechanism using a commonly used and simple Pd catalyst under visible light irradiation. The synthetic utility of this radical strategy was demonstrated through the 1,4-aminoalkylation of dienes. Using this method, a broad range of aliphatic primary, secondary and tertiary carboxylic acids were converted into the corresponding allylic amine motifs. The scalability of these simple and redox-neutral reactions was also demonstrated across a number of examples. Furthermore, this method was successfully applied in the late-stage modification of drugs and natural products. Several complex cascade reactions involving the radical generation of $\pi$-allylpalladium complexes were also developed. Overall, we hope that this radical strategy will be quickly adopted by the synthetic community and used as a complementary method to enable a number of other related cross-coupling and transition-metal catalyzed allylic substitution reactions.

\section{Data availability}

Materials and methods, detailed optimization studies, experimental procedures, mechanistic studies, EPR spectra and NMR spectra are available in the Supplementary Information, or from the corresponding author 
upon reasonable request. The atomic coordinates of the optimized models are provided in Supplementary Data 1. Crystallographic data for compound $\mathbf{2 0}$ is available free of charge from the Cambridge Crystallographic Data Centre under deposition number CCDC 1947092. Copy of the data can be obtained free of charge via https://www.ccdc.cam.ac.uk/structures/.

\section{Acknowledgements}

This work was generously supported by the Alexander von Humboldt Foundation (H.-M.H.), the European Union's Horizon 2020 research and innovation program under the Marie Skłodowska-Curie grant agreement No [843349-H2020-MSCA-IF-2018] (E.S.), and the Deutsche Forschungsgemeinschaft (SFB 858; Leibniz Award). We thank Sina Klabunde for assistance with EPR studies, Bastian Heidrich and Dr. Uta Rodehorst for assistance with XPS studies, Dr. Klaus Bergander for NMR measurements, Dr. Constantin G. Daniliuc for X-ray crystallographic analysis. We also thank Dr. Manuel van Gemmeren, Felix Strieth-Kalthoff, Frederik Sandfort, Dr. Michael J. James, Toryn Dalton, Dr. Jiajia Ma, Dr. Zackaria Nairoukh, Dr. Jian-Heng Ye, Dr. Tunhin Patra, Dr. Chaohuang Chen, Dr. Qiu Sun, Dr. Jun Li and Matthias Freitag (all University of Münster) for helpful discussions.

\section{Author contributions}

H.-M.H. and F.G. directed the project; H.-M.H., M.K., E.S., J.L.S. and F.G. designed the experiments; H.-M.H., M.K. and E.S. performed all the experiments and analyzed all the data. P.M.P performed all the computational studies. H.-M.H. and F.G. wrote the manuscript with contributions from all authors.

\section{Competing interests}

The authors declare no competing interests.

\section{Corresponding Author}

*E-mail: glorius@uni-muenster.de.

\section{ORCID}

Huan-Ming Huang: 0000-0001-9461-6508

Maximilian Koy: 0000-0002-6710-4619

Frank Glorius: 0000-0002-0648-956X

\section{Additional information}

Supplementary information and chemical compound information are available in the online version of the 
paper. Correspondence and requests for materials should be addressed to F.G. (glorius@uni-muenster.de).

\section{References}

1. Trost, B. The atom economy--a search for synthetic efficiency. Science 254, 1471-1477 (1991).

2. Wender, P. A. \& Miller, B. L. Synthesis at the molecular frontier. Nature 460, 197-201 (2009).

3. Young, I. S. \& Baran, P. S. Protecting-group-free synthesis as an opportunity for invention. Nat. Chem. 1, 193-205 (2009).

4. Blakemore, D. C. et al. Organic synthesis provides opportunities to transform drug discovery. Nat. Chem. 10, 383-394 (2018).

5. Trost, B. M. \& Van Vranken, D. L. Asymmetric transition metal-catalyzed allylic alkylations. Chem. Rev. 96, 395-422 (1996).

6. Trost, B. M. \& Crawley, M. L. Asymmetric Transition-Metal-Catalyzed Allylic Alkylations: Applications in Total Synthesis. Chem. Rev. 103, 2921-2944 (2003).

7. Lu, Z. \& Ma, S. Metal-Catalyzed Enantioselective Allylation in Asymmetric Synthesis. Angew. Chem. Int. Ed. 47, 258-297 (2008).

8. Cheng, Q. et al. Iridium-Catalyzed Asymmetric Allylic Substitution Reactions. Chem. Rev. 119, 1855-1969 (2019).

9. Kazmaier, U. Transition Metal Catalyzed Enantioselective Allylic Substitution in Organic Synthesis. (Springer Berlin Heidelberg, 2012).

10. Parisotto, S. \& Deagostino, A. $\pi$-Allylpalladium Complexes in Synthesis: An Update. Synthesis 51, 18921912 (2019).

11. Young, A. J. \& White, M. C. Catalytic Intermolecular Allylic C-H Alkylation. J. Am. Chem. Soc. 130, 1409014091 (2008).

12. Lin, S., Song, C., Cai, G., Wang, W. \& Shi, Z. Intra/Intermolecular Direct Allylic Alkylation via Pd(II)-Catalyzed Allylic C-H Activation. J. Am. Chem. Soc. 130, 12901-12903 (2008).

13. Baeckvall, J. E. Palladium in some selective oxidation reactions. Acc. Chem. Res. 16, 335-342 (1983).

14. Patel, B. A., Dickerson, J. E. \& Heck, R. F. Palladium-catalyzed arylation of conjugated dienes. J. Org. Chem. 43, 5018-5020 (1978).

15. Wu, X. \& Gong, L.-Z. Palladium(0)-Catalyzed Difunctionalization of 1,3-Dienes: From Racemic to Enantioselective. Synthesis 51, 122-134 (2019).

16. Liao, L., Jana, R., Urkalan, K. B. \& Sigman, M. S. A Palladium-Catalyzed Three-Component Cross-Coupling of Conjugated Dienes or Terminal Alkenes with Vinyl Triflates and Boronic Acids. J. Am. Chem. Soc. 133, 5784-5787 (2011).

17. Wu, X. et al. Enantioselective 1,2-Difunctionalization of Dienes Enabled by Chiral Palladium Complex-Catalyzed Cascade Arylation/Allylic Alkylation Reaction. J. Am. Chem. Soc. 137, 13476-13479 (2015).

18. Frisch, A. C. \& Beller, M. Catalysts for Cross-Coupling Reactions with Non-activated Alkyl Halides. Angew. 
Chem. Int. Ed. 44, 674-688 (2005).

19. Prier, C. K., Rankic, D. A. \& MacMillan, D. W. C. Visible Light Photoredox Catalysis with Transition Metal Complexes: Applications in Organic Synthesis. Chem. Rev. 113, 5322-5363 (2013).

20. Yoon, T. P., Ischay, M. A. \& Du, J. Visible light photocatalysis as a greener approach to photochemical synthesis. Nat. Chem. 2, 527-532 (2010).

21. Kärkäs, M. D., Porco, J. A. \& Stephenson, C. R. J. Photochemical Approaches to Complex Chemotypes: Applications in Natural Product Synthesis. Chem. Rev. 116, 9683-9747 (2016).

22. Chuentragool, P., Kurandina, D. \& Gevorgyan, V. Catalysis with Palladium Complexes Photoexcited by Visible Light. Angew. Chem. Int. Ed. 58, 11586-11598 (2019).

23. Okada, K., Okamoto, K., Morita, N., Okubo, K. \& Oda, M. Photosensitized decarboxylative Michael addition through N-(acyloxy)phthalimides via an electron-transfer mechanism. J. Am. Chem. Soc. 113, 9401-9402 (1991).

24. Qin, T. et al. A general alkyl-alkyl cross-coupling enabled by redox-active esters and alkylzinc reagents. Science 352, 801-805 (2016).

25. Fawcett, A. et al. Photoinduced decarboxylative borylation of carboxylic acids. Science 357, 283-286 (2017).

26. Huihui, K. M. M. et al. Decarboxylative Cross-Electrophile Coupling of N -Hydroxyphthalimide Esters with Aryl Iodides. J. Am. Chem. Soc. 138, 5016-5019 (2016).

27. Proctor, R. S. J., Davis, H. J. \& Phipps, R. J. Catalytic enantioselective Minisci-type addition to heteroarenes. Science 360, 419-422 (2018).

28. Ma, J. et al. Synthesis of $\beta$-Substituted $\gamma$-Aminobutyric Acid Derivatives through Enantioselective Photoredox Catalysis. Angew. Chem. Int. Ed. 57, 11193-11197 (2018).

29. Schnermann, M. J. \& Overman, L. E. A Concise Synthesis of (-)-Aplyviolene Facilitated by a Strategic Tertiary Radical Conjugate Addition. Angew. Chem. Int. Ed. 51, 9576-9580 (2012).

30. Mao, R., Frey, A., Balon, J. \& Hu, X. Decarboxylative C( $\left.\mathrm{sp}^{3}\right)-\mathrm{N}$ cross-coupling via synergetic photoredox and copper catalysis. Nat. Catal. 1, 120-126 (2018).

31. Edwards, J. T. et al. Decarboxylative alkenylation. Nature 545, 213-218 (2017).

32. Fu, M.-C., Shang, R., Zhao, B., Wang, B. \& Fu, Y. Photocatalytic decarboxylative alkylations mediated by triphenylphosphine and sodium iodide. Science 363, 1429-1434 (2019).

33. Wang, G.-Z., Shang, R. \& Fu, Y. Irradiation-Induced Palladium-Catalyzed Decarboxylative Heck Reaction of Aliphatic N -(Acyloxy)phthalimides at Room Temperature. Org. Lett. 20, 888-891 (2018).

34. Cheng, W.-M., Shang, R. \& Fu, Y. Irradiation-induced palladium-catalyzed decarboxylative desaturation enabled by a dual ligand system. Nat. Commun. 9, 5215 (2018).

35. Koy, M. et al. Palladium-Catalyzed Decarboxylative Heck-Type Coupling of Activated Aliphatic Carboxylic Acids Enabled by Visible Light. Chem. Eur. J. 24, 4552-4555 (2018).

36. Zhao, W., Wurz, R. P., Peters, J. C. \& Fu, G. C. Photoinduced, Copper-Catalyzed Decarboxylative C-N Coupling to Generate Protected Amines: An Alternative to the Curtius Rearrangement. J. Am. Chem. Soc. 139, 12153-12156 (2017). 
37. Monos, T. M., McAtee, R. C. \& Stephenson, C. R. J. Arylsulfonylacetamides as bifunctional reagents for alkene aminoarylation. Science 361, 1369-1373 (2018).

38. Pitzer, L., Schäfers, F. \& Glorius, F. Rapid Assessment of the Reaction-Condition-Based Sensitivity of Chemical Transformations. Angew. Chemie Int. Ed. 58, 8572-8576 (2019).

39. Banerjee, D., Junge, K. \& Beller, M. Palladium-catalysed regioselective hydroamination of 1,3-dienes: synthesis of allylic amines. Org. Chem. Front. 1, 368 (2014).

40. Plesniak, M. P., Huang, H.-M. \& Procter, D. J. Radical cascade reactions triggered by single electron transfer. Nat. Rev. Chem. 1, 0077 (2017).

41. Sun, X., Chen, J. \& Ritter, T. Catalytic dehydrogenative decarboxyolefination of carboxylic acids. Nat. Chem. 10, 1229-1233 (2018).

42. Parasram, M., Chuentragool, P., Sarkar, D. \& Gevorgyan, V. Photoinduced Formation of Hybrid Aryl Pd-Radical Species Capable of 1,5-HAT: Selective Catalytic Oxidation of Silyl Ethers into Silyl Enol Ethers. J. Am. Chem. Soc. 138, 6340-6343 (2016).

43. Kurandina, D., Parasram, M. \& Gevorgyan, V. Visible Light-Induced Room-Temperature Heck Reaction of Functionalized Alkyl Halides with Vinyl Arenes/Heteroarenes. Angew. Chem. Int. Ed. 56, 14212-14216 (2017).

44. Parasram, M., Chuentragool, P., Wang, Y., Shi, Y. \& Gevorgyan, V. General, Auxiliary-Enabled Photoinduced Pd-Catalyzed Remote Desaturation of Aliphatic Alcohols. J. Am. Chem. Soc. 139, 14857-14860 (2017).

45. Kurandina, D., Rivas, M., Radzhabov, M. \& Gevorgyan, V. Heck Reaction of Electronically Diverse Tertiary Alkyl Halides. Org. Lett. 20, 357-360 (2018).

46. Chuentragool, P. et al. Aliphatic Radical Relay Heck Reaction at Unactivated C( $\left.\mathrm{sp}^{3}\right)-\mathrm{H}$ Sites of Alcohols. Angew. Chem. Int. Ed. 58, 1794-1798 (2019).

47. Ratushnyy, M., Parasram, M., Wang, Y. \& Gevorgyan, V. Palladium-Catalyzed Atom-Transfer Radical Cyclization at Remote Unactivated $\mathrm{C}\left(\mathrm{sp}^{3}\right)-\mathrm{H}$ Sites: Hydrogen-Atom Transfer of Hybrid Vinyl Palladium Radical Intermediates. Angew. Chem. Int. Ed. 57, 2712-2715 (2018).

48. Chuentragool, P., Parasram, M., Shi, Y. \& Gevorgyan, V. General, Mild, and Selective Method for Desaturation of Aliphatic Amines. J. Am. Chem. Soc. 140, 2465-2468 (2018).

49. Wang, G. Z., Shang, R., Cheng, W. M. \& Fu, Y. Irradiation-Induced Heck Reaction of Unactivated Alkyl Halides at Room Temperature. J. Am. Chem. Soc. 139, 18307-18312 (2017).

50. Xing, W., Shang, R., Wang, G.-Z. \& Fu, Y. Visible light-induced palladium-catalyzed ring opening $\beta-H$ elimination and addition of cyclobutanone oxime esters. Chem. Commun. 55, 14291-14294 (2019).

51. Zhou, W. J. et al. Visible-Light-Driven Palladium-Catalyzed Radical Alkylation of C-H Bonds with Unactivated Alkyl Bromides. Angew. Chem. Int. Ed. 56, 15683-15687 (2017).

52. Kancherla, R. et al. Oxidative Addition to Palladium(0) Made Easy through Photoexcited-State Metal Catalysis: Experiment and Computation. Angew. Chem. Int. Ed. 58, 3412-3416 (2019).

53. McMahon, C. M. \& Alexanian, E. J. Palladium-Catalyzed Heck-Type Cross-Couplings of Unactivated Alkyl Iodides. Angew. Chem. Int. Ed. 53, 5974-5977 (2014). 
54. Zou, Y. \& Zhou, J. (Steve). Palladium-catalyzed intermolecular Heck reaction of alkyl halides. Chem. Commun. 50, 3725-3728 (2014).

55. Citterio, A., Arnoldi, A. \& Minisci, F. Nucleophilic character of alkyl radicals. 18. Absolute rate constants for the addition of primary alkyl radicals to conjugated olefins and 1,4-benzoquinone. J. Org. Chem. 44, 2674-2682 (1979).

56. Benn, R. et al. Intermediates in the palladium-catalyzed reactions of 1,3-dienes. 2. Preparation and structure of (.eta.1,.eta.3-octadienediyl)palladium complexes. Organometallics 4, 1945-1953 (1985).

57. Jackstell, R., Grotevendt, A., Andreu, M. G. \& Beller, M. A Practical Palladium-Catalyzed Telomerization for the Synthesis of Functionalized Alcohols. Org. Proc. Res. Dev. 13, 349-353 (2009).

58. Trost, B. M. \& Metzner, P. J. Reaction of olefins with palladium trifluoroacetate. J. Am. Chem. Soc. 102, 3572-3577 (1980).

59. Liu, G., Yin, G. \& Wu, L. Palladium-catalyzed intermolecular aerobic oxidative amination of terminal alkenes: Efficient synthesis of linear allylamine derivatives. Angew. Chem. Int. Ed. 47, 4733-4736 (2008).

60. Reed, S. A. \& White, M. C. Catalytic Intermolecular Linear Allylic C-H Amination via Heterobimetallic Catalysis. J. Am. Chem. Soc. 130, 3316-3318 (2008).

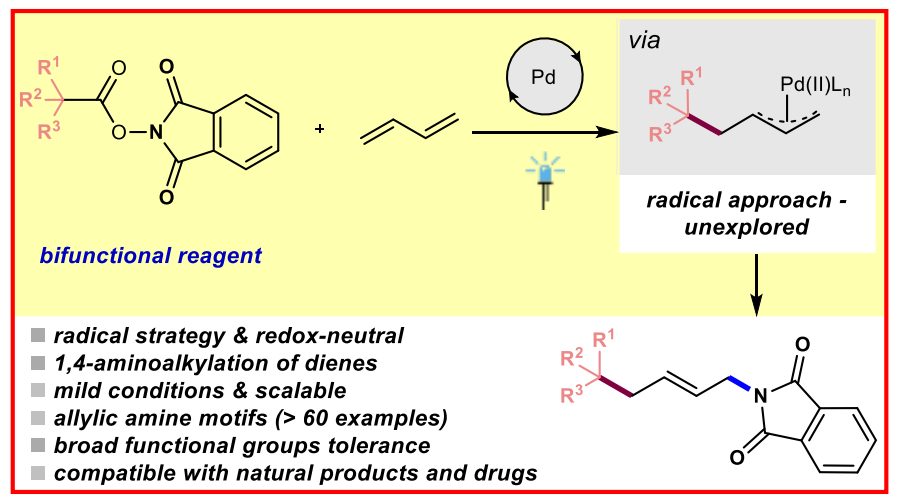

